and anti-inflammatory cytokines maybe a key mechanism for disease progression in RA. IL-25 is reported to play an anti-inflammatory role in autoimmune and inflammatory diseases through the downregulation of Th1 and Th17 cell responses However, the exact role of IL-25 in the pathogenesis of RA remains to be elucidated.

Objectives: Explore the role of IL-25 in the pathogenesis of RA, and provide the scientific evidence of IL-25 relevant biological agent.

Methods: 1.The study was first to analyze the relationship between IL-25 and DAS28 score, CRP and anti-CCP in RA patients. Test IL-1 $\beta$, IL-6, IL-17A, TNF- $\alpha$ and IFN- $\gamma$ through ELISA, then analyze the relationship with IL-25. CD4 ${ }^{+} \mathrm{T}$ cells from PBMCs of the $5 \mathrm{RA}$ patients and $5 \mathrm{HCs}$ were isolated under the manual of EasySep ${ }^{\mathrm{TM}}$ Human $\mathrm{CD}^{+}{ }^{+} \mathrm{T}$ Cell Isolation Kit and stimulated with anti-CD3 plus anti-CD28 in the presence or absence of rhIL-25. 2.CIA model was established to explore the mechanism of IL-25. The expression of Cll specific $\lg G 1$ and $\lg \mathrm{g} 2 \mathrm{a}$, as well as IL-1 $\beta$, IL-6, IL-17A and TNF- $\alpha$ in CIA serum were examined using ELISA assay. The ROR- $\gamma$, T-bet, GATA- 3 mRNA levels in knee joints and spleen were assessed by real-time qPCR analysis. In vitro, $\mathrm{CD}^{+} \mathrm{T}$ cells from spleen were isolated from $\mathrm{CIA}$ mice and cultured with or without recombinant mouse $(\mathrm{rm}) \mathrm{IL}-25$ for $24 \mathrm{~h}$ to control the expression IL-17A. Cells were stimulated with plate-bound anti-CD3 plus anti-CD28 in the presence or absence of recombinant mouse $(\mathrm{rm})$ IL-25 with different dose for $24 \mathrm{~h}$.

Results: 1.The expression of IL-25 were upregulated in the serum and synovial fluid in RA patients. Serum IL-25 levels were positively associated with DAS28 score, ESR and C-reactive protein. However, no significant correlation was observed between serum IL-25 levels, RF and antiCCP. IL-25 serum levels was positively correlated with the concentrations of IL-1 $\beta, \quad$ IL-6, IL-17A and TNF- $\alpha$, respectively. However, no significant correlation was observed between serum IL-25 and IFN- $\gamma$ level. 2. IL-25 can alleviate inflammatory response of $\mathrm{CIA}$ mouse and inhibite IL-17 differentiation. The serum levels of IL-1 $\beta$, IL-6, IL-17A and TNF- $\alpha$ in IL-25treated mice were markedly reduced compared with those in WT mice, except IFN- $\gamma$. RT-qPCR also demonstrated that the transcription levels of IL-17A and ROR- $\gamma$ t were decreased in the synovial tissue of IL-25-treated mice. RmIL-25 treatment significantly inhibited IL-17A production in a dose-dependent manner, and the mRNA level of the key TFs for Th17 cells (ROR- $\gamma \mathrm{t}$ ), as well as IL-17A, were also decreased.

Conclusion: IL-25 is upregulated in the serum and synovial fluid of RA patients. High levels of IL-25 was associated with disease severity and inflammation response in RA patients. IL-25 inhibits CD4+ T-cell activation and differentiation into Th17 cells, without affecting Th1 cells in RA. Systemic administration of IL-25 attenuates arthritis onset and joint damage in CIA mice. IL-25 of multi - target regulation further be used in the clinical treatment of RA.

Disclosure of Interests: None declared

DOI: 10.1136/annrheumdis-2019-eular.8357

\section{THU0044 PRO- AND ANTI-INFLAMMATORY MEDIATORS OF SYSTEMIC INFLAMMATION AND ARTHRITIS IN THE} ELDERLY

Roxana Coras ${ }^{1}$, Rekha Narasimhan ${ }^{1}$, Arthur Kavanaugh ${ }^{1}$, Lourdes Mateo Soria ${ }^{2}$, Oswald Quehenberger ${ }^{1}$, Monica Guma ${ }^{1}$, Melania Martínez-Morillo². ${ }^{1}$ UC San Diego School of Medicine, San Diego, United States of America; ${ }^{2}$ Hospital Germans Trias i Pujol, Rheumatology, Badalona, Spain

Background: Elderly-onset RA (EORA), which is defined as rheumatoid arthritis (RA) with an onset at $>60$ years of age, and polymyalgia rheumatica (PMR) are common rheumatic diseases in the elderly. Eicosanoids are biological lipids that serve a specific role as either activators or suppressors of systemic inflammation and have been involved in the development and progression of arthritis.

Objectives: We hypothesized that eicosanoid-related perturbations are related to arthritic symptoms in the elderly, and that by defining this eicosanoid profile, we might be able to define elements of inflammation pathobiology in this population.

Methods: Arthritis in the Elderly (ARTIEL) is a recent collection cohort with patients older than 60 years of age who have newly diagnosed arthritis. Blood samples were collected from these patients at baseline (pre-treatment) and 3 months after treatment, along with physician and patient outcome measures through 12 months. These patients were also compared with randomly-selected control individuals of the same age and gender. A thorough clinical examination was conducted and patients completed a health assessment questionnaire (HAQ). Disease activity score (DAS)28CRP was also calculated. Serum eicosanoids were determined by mass spectrometry at baseline and after 3 months of treatment and were classified into groups according to their eicosanoid precursors: eicosapen taenoic acid (EPA), docosohexanoic acid (DHA) or arachidonic acid (AA). Data processing and statistical analysis were performed in $R$.

Results: 64 patients (average: 75.15, standard deviation (SD) 6.80) and 18 controls (average: 75.39, SD, 6.04) were analyzed. Of these, 44 were diagnosed with RA and 20 with PMR. At the start of the study, patients had a mean DAS28CRP of $5.72(\mathrm{SD}, 1.05)$ and a mean $\mathrm{HAQ}$ of 1.64 (SD, 0.73). In addition, $84 \%$ of the patients reported scapular pain and $56 \%$ reported pelvic pain at baseline. After three months of treatment, patients had a mean DAS28CRP of 2.38 (SD, 1.23) and a mean HAQ of 0.36 (SD, 0.41). Several eicosanoids, especially anti-inflammatory species derived from EPA and DHA, were significantly downregulated in both RA and PMR patients at the start of the study as compared to normal controls (Figure 1). Three months after treatment, the levels of anti-inflammatory eicosanoid species derived from EPA and DHA went back to normal only in patients who responded to therapy.

Conclusion: These results suggest that certain eicosanoids may be key effectors in arthritis in the elderly and that the imbalance between pro and anti-inflammatory eicosanoids before and after treatment might be related to clinical and therapeutic outcomes in this population.

\section{REFERENCES:}

[1] A systematic review on the role of eicosanoid pathways in rheumatoid arthritis. Hoxha M. Adv Med Sci. 2018 Mar;63(1):22-29.

[2] Kobak S, Bes C. An autumn tale: geriatric rheumatoid arthritis. Therapeutic advances in musculoskeletal disease. 2018 Jan;10(1):3-11. PMID 29290762

[3] Quehenberger O, Dennis EA. The human plasma lipidome. New England Journal of Medicine. 2011 Nov 10;365(19):1812-23. PMID: 22070478

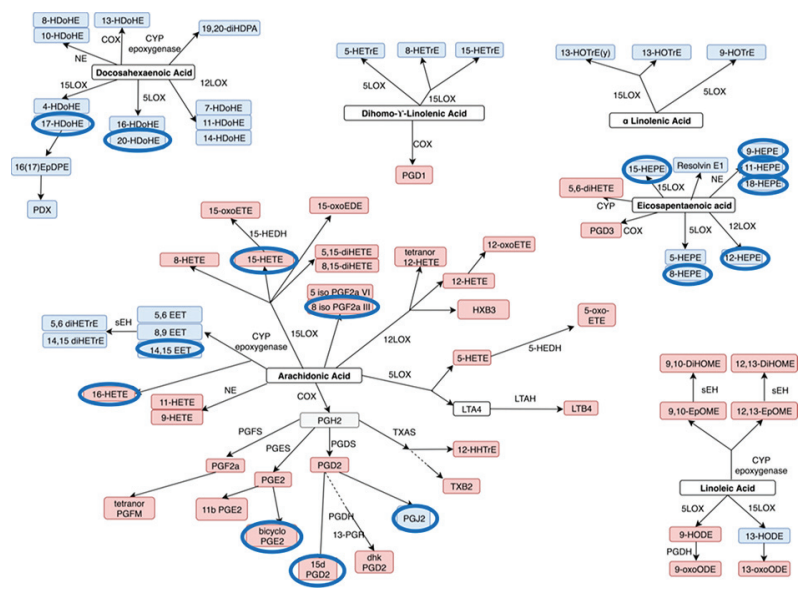

Abstract THU0044 - Figure 1. Anti-inflammatory species derived from EPA and DHA are significantly downregulated in patients at baseline. Pro-inflammatory (in red) and antiinflammatory eicosanoids (in blue) detected in our patients are represented by pathways and origin PUFA. Significant eicosanoids $(p<0.05)$ are circled in blue if downregulated in patients compared to controls at baseline.

Disclosure of Interests: Roxana Coras: None declared, Rekha Narasimhan: None declared, Arthur Kavanaugh Grant/research support from: UCB Pharma, Lourdes Mateo Soria: None declared, Oswald Quehenberger: None declared, Monica Guma: None declared, Melania Martínez-Morillo: None declared

DOI: 10.1136/annrheumdis-2019-eular.2815 